\title{
Doping Mesoporous Materials with Multicolor Quantum Dots
}

\author{
Xiaohu Gao and Shuming Nie* \\ Departments of Biomedical Engineering and Chemistry, Emory University and Georgia Institute of Technology, \\ 1639 Pierce Drive, Suite 2001, Atlanta, Georgia 30322
}

Received: June 30, 2003; In Final Form: July 25, 2003

\begin{abstract}
Rapid and precise doping of mesoporous silica beads has been achieved with luminescent quantum dots, leading to 1-2 orders of magnitude improvements over the results previously achieved with polystyrene latex beads. A surprising finding is that multivalent hydrophobic interactions provide a strong driving force for efficient partitioning and immobilization of surfactant-coated quantum dots in the silica nanopores. The results indicate that the doping levels are so quantitative and precise that at least 30 ratios are distinguishable with two colors of quantum dots and that over 1000 ratios are possible with only three colors. By integrating mesoporous structures and quantum-confined particles, this work opens new possibilities in developing encoded or "smart" nanocomposite materials for multiplexed bioassay, high-throughput screening, and military security applications.
\end{abstract}

\section{Introduction}

Mesoporous silica ${ }^{1-4}$ and semiconductor quantum dots (QDs) $)^{5,6}$ are two distinct classes of nanostructured materials that are under intense study for potential uses in chemical catalysis, chromatographic separations, optoelectronics, and lately for biosensing and biolabeling. ${ }^{7-9}$ Much of the interest in mesoporous silica arises from its ordered structure of nanopores in the size range of $2-100 \mathrm{~nm}$ diameter, ${ }^{10,11}$ which provides a stable and accessible surface for immobilizing a variety of functional molecules and small particles. In parallel, most studies on quantum dots have focused on the quantum confinement effect and their unique size-tunable electronic and optical properties. ${ }^{5,6}$ Combining these two types of nanostructures, for example, incorporation of quantum dots into nanopores, could lead to quantum-dot arrays and novel nanocomposites, but previous research in this area has met with little success. The reason is that most studies have used the nanopores as a reaction template for in situ synthesis, which yielded only poor quantum dots with low quantum yields and broad size distributions. ${ }^{12-16}$ We have solved this problem by using preformed high-quality quantum dots that are coated with a hydrophobic layer of tri-octylphospine oxide (TOPO). By using hydrocarbon-derivatized mesoporous beads of silica, we discuss in this paper the concept of precise QD doping and its potential capabilities and applications.

\section{Experimental Section}

Materials. Core-shell quantum dots (ZnS-capped CdSe) were synthesized according to literature procedures. ${ }^{17,18}$ The resulting quantum dots were coated with a layer of tri- $n$ octylphosphine oxide (TOPO), which was used as a hightemperature coordinating solvent. The QD's fluorescence quantum yields were $\sim 50 \%$ at room temperature, with a size variation of ca. 5\%. Mesoporous materials were synthesized by using pore generating templates such as self-assembled surfac-

* To whom correspondence should be addressed. E-mail address: snie@emory.edu. tants or polymers (called porogens). ${ }^{19,20}$ After synthesis, removal of the templates generated mesosized pores, which were either ordered or random depending on the template structures. In this work, mesoporous silica beads ( $5 \mu \mathrm{m}$ diameter) with pore sizes of 10 or $32 \mathrm{~nm}$ were obtained from Phenomenex (Torrance, $\mathrm{CA})$. The pore surfaces were coated with a monolayer of $\mathrm{Si}-$ $\mathrm{C}_{18} \mathrm{H}_{37}$ (octadecyl, an 18-carbon linear-chain hydrocarbon). These silica materials contained random pores, but the basic principles and methods should be applicable to ordered pores as well.

Doping. Single-color doping was accomplished by mixing porous beads with a controlled amount of quantum dots in an organic solvent such as butanol. In one example, $0.5 \mathrm{~mL}$ of a 4-nM quantum dot solution (chloroform) was mixed with one million porous beads in $2-5 \mathrm{~mL}$ of butanol, yielding a doping level of 1.2 million dots per bead. For the $32-\mathrm{nm}$ pore beads, the doping process was complete in less than $10 \mathrm{~min}$ (no free dots left in solution). For the $10-\mathrm{nm}$ pore beads, more extended times were used to study the doping kinetics. For multiccolor doping, different-colored quantum dots were premixed in precisely controlled ratios. Porous beads were added to an aliquot of this premix solution. Doped beads were isolated by centrifugation and washed three times with ethanol.

Transmission Electron Microscopy (TEM). The doped beads were embedded in a resin (Ted Pella, Redding, CA) and were cut into $60-70 \mathrm{~nm}$ thin sections on an ultramicrotome machine (Leica Ultracut S, Bannockburn, IL). The thin sections were imaged on a Hitachi H-7500 transmission electron microscope operating at $75 \mathrm{k}$ voltage with a magnification of 23000 .

Optical Imaging and Spectroscopy. True-color fluorescence imaging was achieved with an inverted Olympus microscope (IX-70) equipped with a digital color camera (Nikon D1), a broad-band ultraviolet $(330-385 \mathrm{~nm})$ light source $(100-\mathrm{W}$ mercury lamp), and a long-pass interference filter (DM 400, Chroma Tech, Brattleboro, VT). Wavelength-resolved spectra were obtained by using a single-stage spectrometer (SpectraPro 150, Roper Scientific, Trenton, NJ). 
(a)

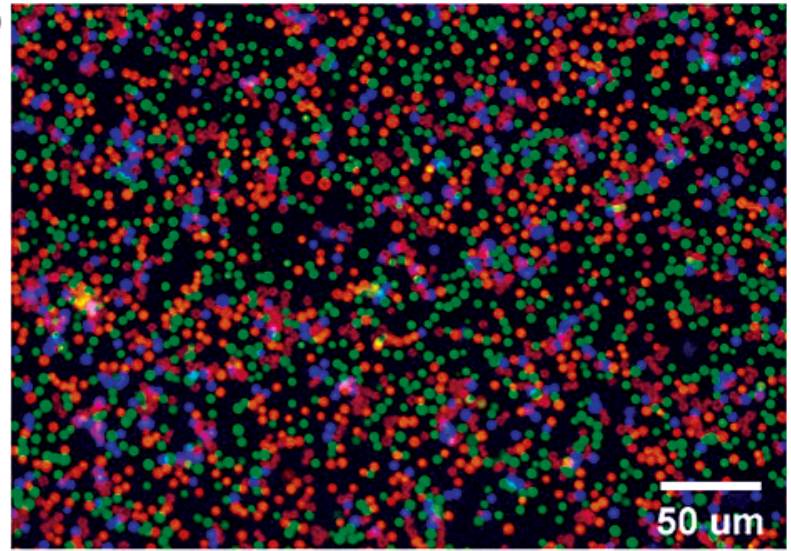

(b)

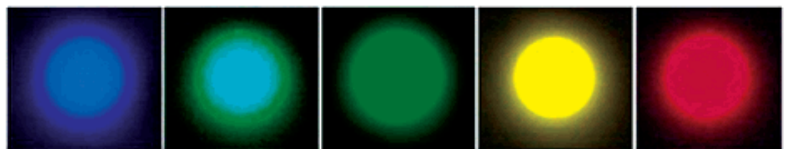

Figure 1. True-color fluorescence images of mesoporous silica beads (5- $\mu \mathrm{m}$ diameter, $32 \mathrm{~nm}$ pore size) doped with single-color quantum dots emitting light at 488 (blue), 520 (green), 550 (yellow), 580 (orange), or $610 \mathrm{~nm}$ (red). (a) Wide-field view of a large population of doped beads that were prepared in batches and then mixed, and (b) enlarged views of single monochromatic beads. Each bead contains up to 2 million dots of the same color.

\section{Results and Discussion}

Figure 1 shows true-color fluorescence images of QD-doped silica beads ( $5.0 \pm 0.3 \mu \mathrm{m}$ diameter) with an average pore size of $32 \pm 4 \mathrm{~nm}$. These beads are considered "monochromatic" because they were prepared by using single-color quantum dots, and were mixed and spread on a glass surface for fluorescence imaging. Although only a single light source (a near-UV lamp) was used for excitation, all of the doped beads were observed and were clearly distinguishable. It is worth noting that simultaneous excitation of multiple emission colors is a unique property of quantum dots and is not possible with organic dyes or lanthanide compounds. The QD-doped beads are remarkably bright and can be recorded by using a digital color camera and a mercury lamp. Quantitative measurements indicate that these mesoporous beads are ca. 50-100 times brighter than the QDtagged latex beads reported previously ${ }^{21}$ and are more than 30 times brighter than nonporous polystyrene beads of similar sizes.

A key factor contributing to the improved brightness is the large surface area $\left(150 \mathrm{~m}^{2}\right.$ per gram for the 32 -nm pore beads), about 50-100 times larger than the surface area of nonporous beads. Also, the quantum dots are able to penetrate deeply into the mesoporous beads, as shown by the remarkably uniform fluorescence across the bead interior (enlarged images in Figure $1 b$, and cross sectional data in Figure 3b). In comparison, the penetration depths of quantum dots into polystyrene beads do not exceed $5 \%$ of the bead's radius as measured by transmission electron microscopy, even under extensive swelling conditions (R. Bailey and S. Nie, unpublished data). Confocal fluorescence imaging shows a clear ring pattern confirming that the quantum dots are primarily located on the surface or the adjacent layer (data not shown). This is not surprising because previous research has shown that even hydrogels such as Sepharose do not allow penetration of 30-nm colloidal gold beyond a $0.1-$ $0.2 \mu \mathrm{m}$ boundary layer. ${ }^{22}$

To further examine the effect of pore size on doping kinetics, we compared the time-dependent fluorescence signals for two types of silica beads, one with a pore size of $32 \mathrm{~nm}$ and the

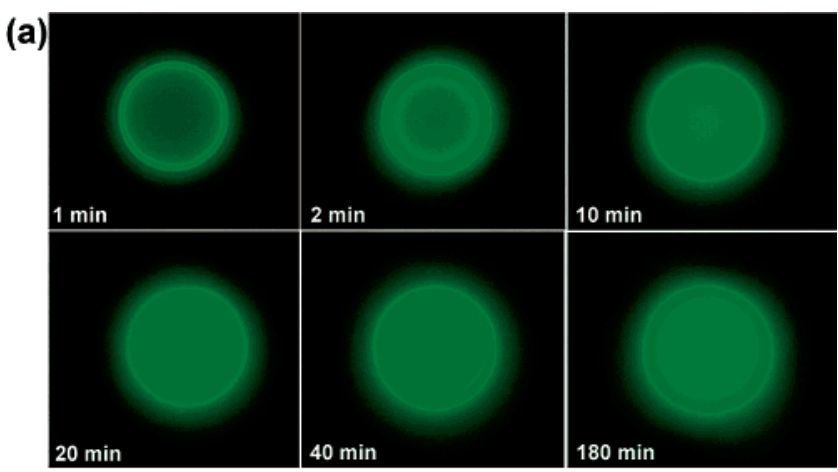

(b)

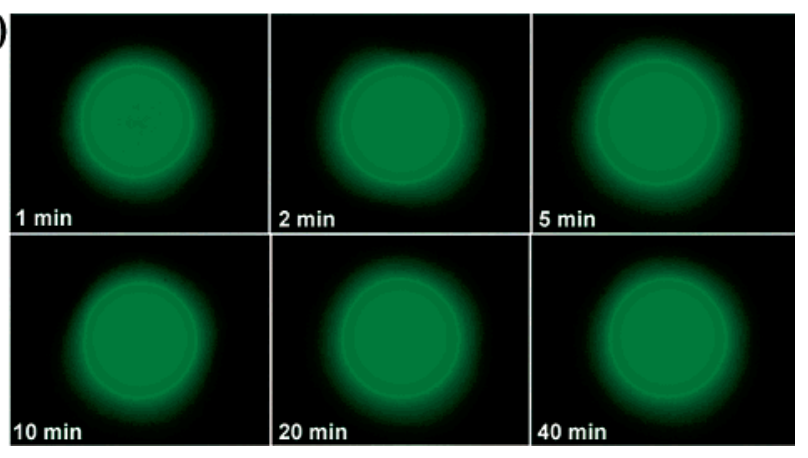

Figure 2. Time-dependent fluorescence images showing the effects of pore size on doping kinetics. (a) 10-nm pore beads and (b) 32-nm pore beads. Except for the pore size, all other bead properties (size, material, and surface chemistry) and the data acquisition conditions (excitation wavelength, intensity, and exposure time) were the same.

other $10 \mathrm{~nm}$ (Figure 2). The results reveal that doping is remarkably fast for the 32-nm pore beads, achieving nearly uniform QD distribution in 1 min and saturation in less than 5 $\min$. For the 10-nm pore beads, however, a blurred ring structure is observed from $1-60 \mathrm{~min}$, indicating that doping primarily occurs on the bead surface. This uneven structure disappears after $\sim 3 \mathrm{~h}$, but the fluorescence intensity is not saturated until $10 \mathrm{~h}$ later. This comparison suggests that the $32-\mathrm{nm}$ pores are large enough for rapid QD diffusion, but the 10-nm pores significantly reduce QD diffusion and the doping kinetics. Considering the thickness of the hydrocarbon coating $(2 \mathrm{~nm}$ on all sides), the open space in a $10-\mathrm{nm}$ pore would be reduced to $6 \mathrm{~nm}$, roughly the dimension of a 3-4-nm QD plus a 1-nm TOPO capping layer.

Prepared as $60-70 \mathrm{~nm}$ thin sections, the doped beads were examined by both transmission electron microscopy (TEM) and wide-field optical imaging (Figure 3). The TEM data showed a highly porous structure but could not resolve single nanopores or quantum dots at low resolutions. Optical imaging revealed uniform fluorescence signals across the diameters of single beads, although random "hot spots" and bead-to-bead variations were observed. Overall, this cross-sectional study unambiguously demonstrates deep penetration of quantum dots into the bead's interior.

Inside the pores, quantum dots are confined via hydrophobic interactions between the hydrocarbon and TOPO molecules, and doping occurs when the hydrocarbon and surfactant molecules (both are conformationally flexible) insert into each other in a stable, interdigitated configuration (Figure 4a). Assuming a surface density of 1-2 hydrocarbon or TOPO molecules per square nanometer (typical for monolayer coverage), we estimate that about 10 TOPO molecules on a single dot can react with roughly the same number of hydrocarbon molecules on the nanopore wall (based on a sterically allowed solid angle of 0.5 $\pi$ for molecular interactions). As widely known in chemistry 


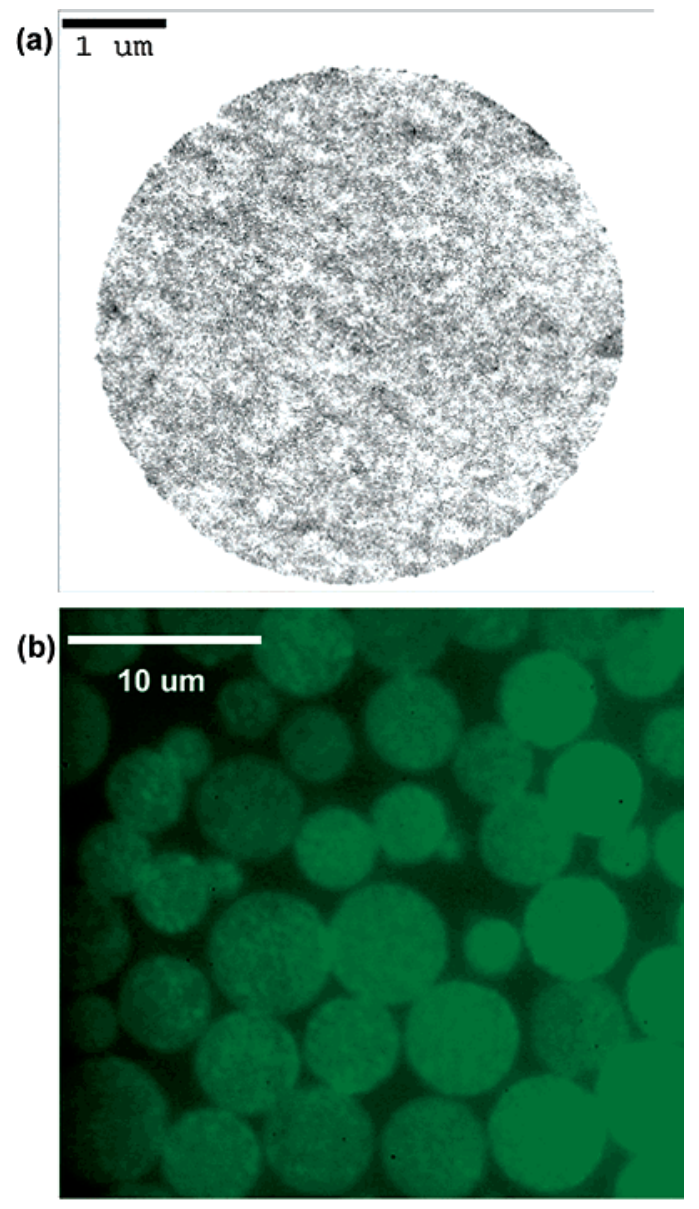

Figure 3. Cross sectional imaging of QD-doped silica beads cut into 60-70 $\mathrm{nm}$ thin sections. (a) TEM image of a $5 \mu \mathrm{m}$ bead showing a highly porous internal structure. (b) Wide-field fluorescence imaging of a thin section showing deep penetration of quantum dots into the bead's interior. Because the cross section of a bead depends on where it is cut, randomly embedded beads of the same size would show different cross sections, as observed in the wide-field optical image.

and biology, ${ }^{23}$ weak multivalent interactions can lead to stable complexes with very slow dissociation rates. Thus, the quantum dots are expected to be firmly immobilized in the pores and are stable against dissociation or leaching. Indeed, no QD leakage was observed when the doped beads were exposed overnight to water or polar organic solvents (e.g., ethanol, acetone, acetonitrile, and DMF) (Figure 4b), yielding a flat line of background (supernatant) fluorescence intensity with time. In chloroform or other nonpolar solvents, however, QDs were observed to slowly leach out of the beads.

A further question is whether the quantum dots exist as dispersed single particles or as aggregates in the nanopores. Aggregation could cause problems such as spectral broadening, self-quenching, and wavelength shifting. The results indicate that the optical properties of quantum dots are nearly identical, either when they are dispersed in organic solvents or are immobilized in silica beads (Figure 4c). One can thus conclude that the QDs are spatially isolated from each other inside the beads, similar to that of organic dyes dispersed in mesoporous silica. $^{24-26}$ By using two independent measures (single-dot spectroscopy and bulk concentration measurement) reported previously, ${ }^{21}$ we estimate that the numbers of quantum dots per bead are as large as 2 million (depending on the pore size), corresponding to ca. $5 \%$ surface coverage or occupancy rate. This condition is still far from surface saturation and is thus favorable for quantum-dot dispersion inside the beads. Quantita- (a)

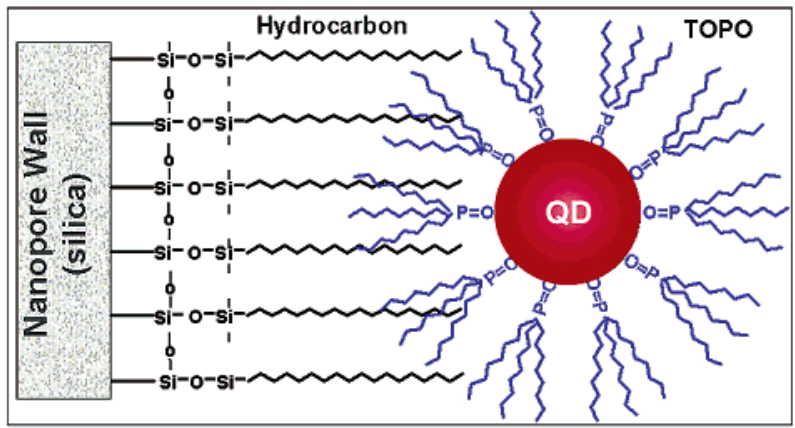

(b)

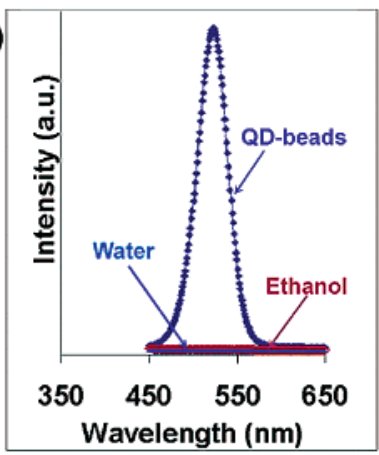

(c)

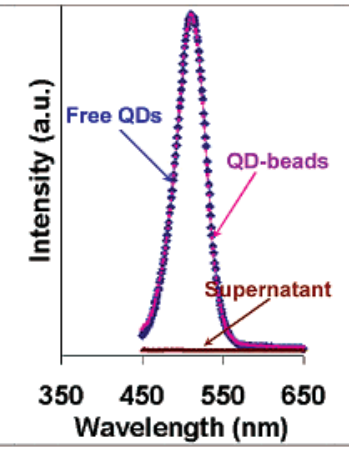

Figure 4. Doping mechanism and optical properties of quantum dots embedded in mesoporous silica. (a) Schematic illustration of multivalent hydrophobic interactions between the surfactant (TOPO) molecules on the QD surface and the $\mathrm{C}-18$ hydrocarbon molecules on the pore walls. (b) Fluorescence data showing the stability of the doped beads and the absence of quantum dots leaching into water or ethanol after overnight exposure at room temperature. Beads were removed by centrifugation, and supernatants (water or ethanol) were measured on a fluorescence spectrometer, which was sensitive to even trace amounts of leached quantum dots. All fluorescence spectra were plotted on the same intensity scale. (c) Fluorescence data showing identical optical properties of quantum dots dispersed in solution or immobilized in mesoporous silica beads. The supernatant curve showed no quantum dots in the solution (nearly $100 \%$ incorporation into the beads).

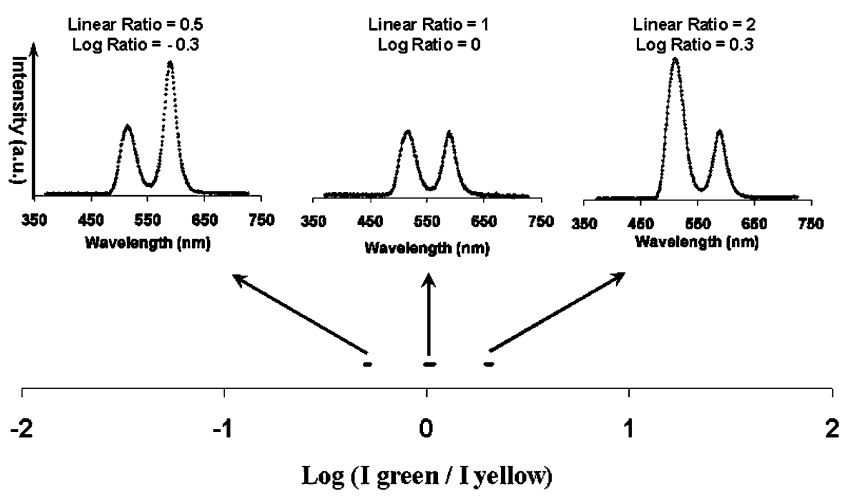

Figure 5. Ratiometric doping results using two QD colors and two intensity levels. Top: actual fluorescence spectra showing three intensity ratios of 1:2, 1:1, and 2:1 at emission wavelengths of 510 and $580 \mathrm{~nm}$. Bottom: scatter plot of the intensity ratios (log scale) based on 30 data points (single-bead measurements) at each ratio.

tive measurement further reveals that essentially no quantum dots are left in the free solution, due to nearly 100\% QD incorporation into the beads. This is especially important for multicolor doping, in which the amounts of different quantum dots must be controlled precisely.

Figure 5 shows quantitative doping results obtained from twocolor encoded beads. Using two intensities, there are three unique intensity ratios $(1: 2,1: 1$, and $2: 1)$. These ratios are remarkably robust in a given class of doped beads, although the absolute intensities could vary considerably from bead to 
bead (because of variations in bead size and instrumental aligment). As can be seen from the tight scatter plot, the standard deviations for these intensity ratios are less than $2.0 \%$. This high level of reproducibility allows 20 intensity ratios to be distinguished within one log along the $x$ axis and 40 ratios within two logs. Thus, in a 3-color doping scheme (yielding two independent ratios), $400(20 \times 20)$ doping combinations are possible with $\pm 1 \log$, and up to $1600(40 \times 40)$ combinations are possible with \pm 2 logs. As reported by Weiss and co-workers for biomolecular imaging, ${ }^{27}$ ratiometric measurements are much more reliable than absolute intensities because the ratio values are not affected by simultaneous drifts or fluctuations of the individual signals. This advantage will help the development of microdevices and algorithms for reading the doped beads at high speeds and accuracies.

In conclusion, we have reported a simple strategy for rapid and precise doping of mesoporous silica materials with multicolor quantum dots. In comparison with other doping or optical encoding technologies, ${ }^{28-36}$ the use of mesostructured silica provides an unprecedented level of doping control, better than $2 \%$ reproducibility, and low production costs. For potential applications in combinatorial or multiplexed assays, the doped beads could be linked to biomolecules such as oligonucleotides, peptides, or antibodies. ${ }^{8,21}$ Both the outer bead surface and the internal pore surface might be used because the mesoporous structure allows rapid diffusion and fast response. In addition, QD-doped mesoporous fibers and thin films will have applications in chemical/biological sensing, quantum-dot lasers, and integrated optoelectronics. Furthermore, the multivalent doping method can be extended to electrostatic, ligand-receptor, and metal ion chelating interactions for doping nanoporous materials with various molecules and nanoparticles. ${ }^{37,38}$

Acknowledgment. The authors acknowledge Dr. Hong Yi at Emory University School of Medicine for TEM measurements. This work was supported by the National Institutes of Health (R01 GM60562) and the Coulter Translational Research Program at Georgia Tech and Emory University.

\section{References and Notes}

(1) Davis, M. E. Nature 2002, 417, 813-821.

(2) Scott, B. J.; Wirnsberger, G.; Stucky, G. D. Chem. Mater. 2001, $13,3140-3150$

(3) Schuth, F.; Schmidt, W. Adv. Mater. 2002, 14, 629-638.

(4) Lin, H.-P.; Mou, C.-Y. Acc. Chem. Res. 2002, 35, 927-935.

(5) Alivisatos, A. P. Science 1996, 271, 933-937.

(6) Murray, C. B.; Kagan, C. R.; Bawendi, M. G. Annu. Rev. Mater. Sci. 2000, 30, 545-610.

(7) Murphy, C. J. Anal. Chem. 2002, 74, 520A-526A

(8) Chan, W. C. W.; Maxwell, D. J.; Gao, X.; Bailey, R. E.; Han, H. Y.; Nie, S. Curr. Opin. Biotechnol. 2002, 13, 40-46.

(9) Chan, W. C. W.; Nie, S. M. Science 1998, 281, 2016-2018.
(10) Kresge, C. T.; Leonowicz, M. E.; Roth, W. J.; Vertuli, J. C.; Beck, J. S. Nature 1992, 359, 710-712.

(11) Zhao, Y.; Feng, J.; Huo, Q.; Melosh, N.; Fredrickson, G. H.; Chmelka, B. F.; Stucky, G. D. Science 1998, 279, 548-552.

(12) Parala, H.; Winkler, H.; Kolbe, M.; Wohlfart, A.; Fischer, R. A.; Schmechel, R.; von Seggern, H. Adv. Mater. 2000, 12, 1050-1055.

(13) Winkler, H.; Birkner, A.; Hagen, V.; Wolf, I.; Schmechel, R.; von Seggern, H. Adv. Mater. 1999, 11, 1444-1448.

(14) Agger, J. R.; Anderson, M. W.; Pemble, M. E.; Terasaki, O.; Nozue, Y. J. Phys. Chem. B 1998, 102, 3345-3353.

(15) Hirai, T.; Okubo, H.; Komasawa, I. J. Phys. Chem. B. 1999, 103 , $4228-4230$

(16) Xu, W.; Liao, Y.; Akins, D. L. J. Phys. Chem. B 2002, 106, 1112711131

(17) Dabbousi, B. O.; Rodriguez-Viejo, J.; Mikulec, F. V.; Heine, J. R.; Mattoussi, H.; Ober, R.; Jensen, K. F.; Bawendi, M. G. J. Phys. Chem. $B$ 1997, 101, 9463-9475.

(18) Peng, Z. A.; Peng, X. G. J. Am. Chem. Soc. 2001, 123, 183-184.

(19) Wang, Q. C.; Hosoya, K.; Svec, F.; Frechet, J. M. Anal. Chem. 1992, 64, 1232-1238.

(20) Iler, R. K.; Kirkland, J. J. Process for manufacture of macroporous microspheroids. US Patent No. 4,105,426, 1978.

(21) Han, M. Y.; Gao, X. H.; Su, J. Z.; Nie, S. Nat. Biotechnol. 2001, $19,631-635$.

(22) Kehle, T.; Herzog, V. In: Colloidal Gold - Principles, Methods, and Applications; Hayat, M. A., Ed.; Academic Press: New York, 1991; Vol. 3, Chapter 6, pp 117-137.

(23) Mammen, M.; Choi, S.-K.; Whitesides, G. M. Angew. Chem. 1998, 37, 2754-2794

(24) Yang, P.; Wirnsberger, G.; Huang, H. C.; Cordero, S. R.; McGehee, M. D.; Scott, B.; Deng, T.; Whitesides, G. M.; Chmelka, B. F.; Buratto, S. K.; Stucky, G. D. Science 2000, 287, 465-67.

(25) Wirnsberger, G.; Stucky, G. D. Chem. Mater. 2000, 12, 25252627.

(26) Marlow, F.; McGehee, M. D.; Zhao, D.; Chmelka, B. F.; Stucky, G. D. Adv. Mater. 1999, 11, 632-636.

(27) Deniz, A. A.; Laurence, T. A.; Dahan, M.; Chemla, D. S.; Schultz,

P. G.; Weiss, S. Annu. Rev. Phys. Chem. 2001, 52, 233-253.

(28) Walt, D. R. Curr. Opin. Chem. Biol. 2002, 6, 689-695.

(29) Nicewarner-Pena, S. R.; Freeman, R. G.; Reiss, B. D.; He, L.; Pena, D. J.; Walton, I. D.; Cromer, R.; Keating, C. D.; Natan, M. J. Science 2001, 294, 137-141.

(30) Cunin, F.; Schmedake, T. A.; Link, J. R.; Li, Y. Y.; Koh, J.; Bhatia, S. N.; Sailor, M. J. Nat. Mater. 2002, 1, 39-41.

(31) Braeckmans, K.; de Smedt, S.; Roelant, C.; Leblans, M.; Pauwels, R.; Demeester, J. Nat. Mater. 2003, 2, 169-193.

(32) Dejneka, M. J.; Streltsov, A.; Pal, S.; Frutos, A. G.; Powell, C. L.; Yost, K.; Yuen, P. K.; Muller, U.; Lahiri, J. Proc. Natl. Acad. Sci. U.S.A 2003, 100, 389-393.

(33) Grondahl, L.; Battersby, B. J.; Bryant, D.; Trau, M. Langmuir 2000 16, 9709-9715.

(34) Gaponik, N.; Radtchenko, I. L.; Sukhorukov, G. B.; Weller, H.; Rogach, A. L. Adv. Mater. 2002, 14, 879-882.

(35) Konya, Z.; Puntes, V. F.; Kiricsi, I.; Zhu, J.; Alivisatos, A. P.; Somorjai, G. A. Nano Lett. 2002, 2, 907-910

(36) Konya, Z.; Puntes, V. F.; Kiricsi, I.; Zhu, J.; Ager, III, J. W.; Ko, M. K.; Frei, H.; Alivisatos, A. P.; Somorjai, G. A. Chem. Mater. 2003, 15, $1242-1248$

(37) Lee, S. B.; Michell, D. T.; Trofin, L.; Nevanen, T. K.; Soderlund, H.; Martin, C. R. Science 2002, 296, 2198-2200. 1540. 